\title{
Reversing anticoagulant effects of novel oral anticoagulants: role of ciraparantag, andexanet alfa, and idarucizumab
}

REVIEW

This article was published in the following Dove Press journal:

Vascular Health and Risk Management

17 February 2016

Number of times this article has been viewed

Tiffany $\mathrm{Y} \mathrm{Hu}{ }^{\prime}$

Vaibhav R Vaidya ${ }^{2}$

Samuel J Asirvatham ${ }^{2,3}$

'Mayo Medical School, '2Division of Cardiovascular Diseases, Department of Internal Medicine, ${ }^{3}$ Department of Pediatrics and Adolescent Medicine, Mayo Clinic, Rochester, MN, USA

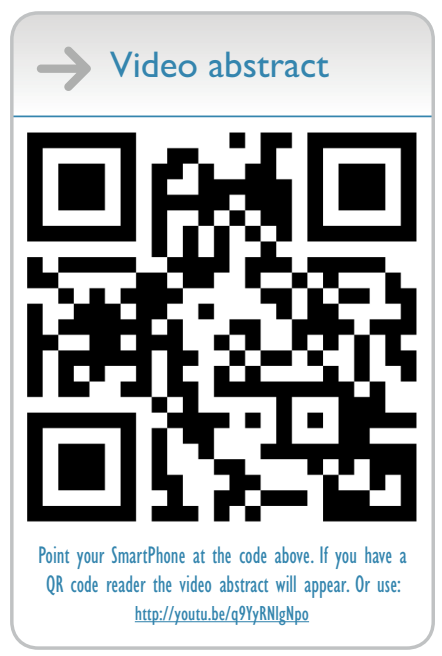

Correspondence: Samuel J Asirvatham Department of Pediatrics and Adolescent Medicine, Mayo Clinic, 200 First Street SW, Rochester, MN 55905, USA

Tel +l 5072933376

Fax + I 5072552550

Email asirvatham.samuel@mayo.edu

\begin{abstract}
Novel oral anticoagulants (NOACs) are increasingly used in clinical practice, but lack of commercially available reversal agents is a major barrier for mainstream use of these therapies. Specific antidotes to NOACs are under development. Idarucizumab (aDabi-Fab, BI 655075) is a novel humanized mouse monoclonal antibody that binds dabigatran and reverses its anticoagulant effect. In a recent Phase III study (Reversal Effects of Idarucizumab on Active Dabigatran), a $5 \mathrm{~g}$ intravenous infusion of idarucizumab resulted in the normalization of dilute thrombin time in $98 \%$ and $93 \%$ of the two groups studied, with normalization of ecarin-clotting time in $89 \%$ and $88 \%$ patients. Two other antidotes, andexanet alfa (PRT064445) and ciraparantag (PER977) are also under development for reversal of NOACs. In this review, we discuss commonly encountered management issues with NOACs such as periprocedural management, laboratory monitoring of anticoagulation, and management of bleeding. We review currently available data regarding specific antidotes to NOACs with respect to pharmacology and clinical trials.
\end{abstract}

Keywords: novel oral anticoagulant, dabigatran, idarucizumab, reversal

\section{Introduction}

For decades, vitamin $\mathrm{K}$ antagonists such as warfarin were the only oral agents available for long-term anticoagulation. Warfarin's variable bioavailability and drug-drug interactions complicate achievement of therapeutic anticoagulation and necessitate regular monitoring. The advent and increasing clinical use of non-vitamin $\mathrm{K}$ or novel oral anticoagulants (NOACs) is changing the status quo. Direct factor Xa inhibitors (apixaban and rivaroxaban) and direct thrombin inhibitors (dabigatran) are approved for stroke prevention in atrial fibrillation and prophylaxis and treatment of venous thromboembolism (VTE) in the US and Europe, though dabigatran is not yet approved for VTE prophylaxis in the US. ${ }^{1-6}$ More recently, another direct factor Xa inhibitor, edoxaban, was approved for stroke prevention in atrial fibrillation and VTE treatment and prevention of VTE recurrence in the US and Europe. ${ }^{7-11}$ Of note is the latest investigational NOAC, the direct factor Xa inhibitor Betrixaban, has the lowest renal clearance and hepatic metabolism and longest half-life among the NOACs. ${ }^{12,13}$ It has undergone Phase II trials for stroke prevention in atrial fibrillation and VTE prevention, and it is currently undergoing Phase III investigation for extended thromboprophylaxis for high-risk patients. Compared to warfarin, NOACs have decreased bleeding risk with non-inferior efficacy in patients with atrial fibrillation. ${ }^{1-3,7,14}$ A meta-analysis of 12 randomized controlled trials involving 102,607 patients demonstrated the superior safety of NOACs compared to warfarin for the treatment of VTE or atrial fibrillation. ${ }^{15}$ 
NOACs were associated with lower rates of major bleeding, intracranial bleeding, clinically relevant but non-major bleeding, and total bleeding. ${ }^{15,16}$

Unlike warfarin, which may be reversed with fresh frozen plasma and vitamin $\mathrm{K}$, there are no approved reversal agents for NOACs. Despite the relative safety of NOACs with respect to hemorrhagic complications, these complications do occur, and up until idarucizumab's recent US Food and Drug Administration (FDA) approval, there existed an unmet need for dedicated reversal agents. In this article, we discuss general management strategies for bleeding complications among patients receiving NOACs and available specific antidotes for NOACs with a focus on idarucizumab - a monoclonal antibody designed to reverse anticoagulation with dabigatran.

\section{Management problems with NOACs}

Given their relatively recent introduction to clinical use, there are fewer data regarding the management of NOACs. There are three areas of uncertainty with respect to management of NOACs: perioperative management, laboratory monitoring of anticoagulation, and management of bleeding. Perioperative management of NOACs can be challenging due to lack of data from large randomized studies. While the international normalized ratio (INR) is routinely used to monitor warfarin and activated partial thromboplastin time (aPTT) used to monitor heparin, monitoring of NOACs with laboratory tests is less clear. A third area of uncertainty is management of bleeding complications in patients receiving NOACs. Despite limited data, there is more clinical experience with warfarin to form guidelines for perioperative management, monitoring, and reversal with vitamin $\mathrm{K}$ or plasma factors. ${ }^{4}$ Such guidelines for NOACs are currently unavailable.

\section{Management of periprocedural anticoagulation with NOACs}

In general, surgeries with low risk of bleeding can be safely performed on therapeutic anticoagulation. ${ }^{17-19}$ For surgeries with moderate to severe risk of bleeding, the risk of bleeding must be weighed against the risk of thrombosis off anticoagulation, and the decision to stop the NOAC must be individualized. Our approach to periprocedural management of NOACs is discussed next. ${ }^{20}$

The timing of cessation of NOAC prior to surgery depends on the half-life of the agent, procedure-specific bleeding risks, and renal function of the patient. In general, Factor
Xa inhibitors must be stopped at least 24-48 hours prior to surgery with moderate bleeding risk and 48-72 hours prior to surgery with high bleeding risk. Dabigatran may be stopped $>72$ hours prior to surgeries with moderate bleeding risk. Patients with impaired renal function should have NOACs stopped earlier. Timing of reinitiation of NOACs following surgery depends on achievement of adequate hemostasis. For moderate risk surgeries, NOACs may be restarted 24 hours after the procedure if hemostasis is achieved. For higher risk surgeries, a 48-hour period may be more appropriate. Unlike warfarin, the time to effective anticoagulation is within hours of first administration for all NOACs. ${ }^{6,17-19,21}$

Phase II trials regarding NOAC efficacy in thromboprophylaxis suggest that a reduced dose 6-12 hours after surgery may be a safe and effective time to begin prophylaxis. ${ }^{22}$ In the absence of studies specifically addressing this issue, a general time frame of 24 hours for resumption of rivaroxaban, apixaban, and edoxaban may be appropriate until more data are available.

Recently, the timeline for dabigatran discontinuation and resumption perioperatively has been evaluated prospectively. The timing of the last dose of dabigatran before the procedure is less conservative compared to our approach, and resumption of NOAC post-procedure is overall earlier as well. ${ }^{23}$

\section{Monitoring with coagulation studies}

Routine coagulation studies including INR and aPTT may not accurately reflect the level of anticoagulation among patients receiving NOACs. In bleeding patients, however, specific laboratory values of anticoagulation can be helpful in guiding management decisions. INR is not recommended for monitoring of any NOAC agent. The aPTT is elevated in patients taking dabigatran and edoxaban, but there is no clear dose-response relationship. ${ }^{17,24}$

Specialized assays including thrombin time (TT), dilute thrombin time (dTT), and ecarin-clotting time (ECT) are currently used in research studies. The TT is a very sensitive assay for dabigatran activity, and it can be useful for ensuring complete elimination of dabigatran prior to high-risk elective surgery. ${ }^{20} \mathrm{~A}$ quantifiable dose-response relationship is present for dabigatran and ECT and dTT, but these assays are not commercially available at most institutions. For apixaban and rivaroxaban, the anti-factor Xa chromogenic assay yields results consistent with dose-response, but results may not be available in a timely fashion. There remains an unmet need for accurate, commercially available measures of anticoagulation for NOACs that can be performed expeditiously in emergency settings. 


\section{General management of bleeding in patients on NOACs}

The risk of life-threatening bleed remains a concern with any anticoagulation therapy. In anticoagulated patients who present with bleeding, several factors and considerations affect management decisions, such as supportive care, withdrawal of the offending agent, and use of specific and non-specific hemostatic therapies:

- As with any bleeding event, the site, rate, volume of blood loss, and vital signs should be used to risk stratify the patient.

- Serial complete blood counts may provide information on the rate and volume of bleeding, with the caveat that in massive hemorrhage, the hemoglobin may not immediately reflect the severity of blood loss.

- Time of last anticoagulant dose, the potential for overdose, and possible medication interactions.

- Altered anticoagulant pharmacokinetics - malabsorption of NOACs and/or vitamin $\mathrm{K}$ and hepatic and renal dysfunction.

- Consideration of concomitant etiologies of bleeding, such as thrombocytopenia or disseminated intravascular coagulation.

In the absence of US FDA-approved NOAC antidotes and prospective data to guide bleed management in the setting of NOAC use, several hemostatic therapies have been described. There is little data on the efficacy of these in NOAC-associated bleeding. Strategies can be categorized as follows:

- Alter pharmacokinetics: reduce the absorption or remove from circulation

- Antifibrinolytic agents-tranexamic acid and $\varepsilon$-aminocaproic acid

- Therapy with plasma factors: prothrombin complex concentrates (PCCs), fresh frozen plasma, and cryoprecipitate

- Specific antidotes: ciraparantag (PER977), andexanet alfa, and idarucizumab

\section{Minor bleeds}

Supportive measures such as mechanical compression and discontinuation of anticoagulation (if the bleed risk outweighs the thrombosis risk) is typically most appropriate in minor bleeds such as epistaxis and ecchymosis. ${ }^{17-19,21}$

\section{Major bleeding}

In cases of major bleeding, the offending NOAC agent must be stopped. Supportive measures such as intravenous hydration, administration of packed red blood cells, and platelet transfusions for thrombocytopenia are essential. Mechanical or surgical compression is invaluable in controlling the source of the bleeding. In addition, the following strategies may be used to achieve hemostasis.

\section{Altering pharmacokinetics of NOACs}

For major bleeding associated with apixaban and dabigatran, administration of activated charcoal may reduce continued absorption of the anticoagulant (Table 1). Limited data shows that charcoal administered up to 6 hours after apixaban reduces its plasma concentration, with best effects when administered within 2 hours from ingestion. ${ }^{25}$ Similarly, activated charcoal may be useful in decreasing dabigatran levels when given within 2-3 hours of ingestion. Limited ex vivo data but no human data are available for dabigatran. ${ }^{26}$ Although there are no specific studies available for rivaroxaban or edoxaban, activated charcoal may be efficacious for these agents too. Given the limited timeframe for utility, charcoal may be most useful in instances of overdose.

Hemodialysis is a possible treatment for dabigatranassociated bleeding, given dabigatran's low protein-bound state and lipophilic profile. ${ }^{18}$ Rivaroxaban and apixaban are more highly protein-bound and thus are unlikely to be successfully dialyzed. It is unclear if edoxaban may be removed by dialysis. Small case series and case reports have described achieving hemostasis in patients using intermittent hemodialysis and continuous renal replacement therapy, sometimes in tandem with supportive blood transfusions. ${ }^{27}$ Dialysis is challenging in cases with major bleeding and hemodynamic instability, and is most likely to be of the greatest utility in instances of dabigatran overdose or emergent need for surgery in otherwise hemodynamically stable patients.

\section{Antifibrinolytic agents}

In the case of major bleeding, antifibrinolytic agents such as tranexamic and $\varepsilon$-aminocaproic acid may be used. Their use has been best described in the operating room to decrease blood loss and in the postoperative setting, with a superior safety profile to aprotinin, an agent no longer available in the US. ${ }^{17,24}$ Although the efficacy of these agents in case of NOAC-associated bleeding is unknown, these are inexpensive agents with a favorable safety profile.

\section{Therapy with plasma factors}

The use of concentrated coagulation factors in emergency, life-threatening bleeds to prevent imminent death has been 
Table I General management of bleeding in patients on novel oral anticoagulant therapy

\begin{tabular}{|c|c|c|c|c|}
\hline & Dabigatran & Rivaroxaban & Apixaban & Edoxaban \\
\hline Minor bleeding & \multicolumn{4}{|c|}{ Mechanical compression, interrupt anticoagulation if appropriate } \\
\hline Major bleeding & \multicolumn{4}{|c|}{ IV fluids, correct anemia, thrombocytopenia, source control. Stop agent } \\
\hline Activated charcoal & Yes* & Unknown & Yes* & Unknown \\
\hline Hemodialysis & Yes $\S$ & No & No & Unknown \\
\hline Antifibrinolytics & \multicolumn{4}{|c|}{ Tranexamic acid and $\varepsilon$-aminocaproic acid ${ }^{\infty}$} \\
\hline Plasma factors & \multicolumn{4}{|c|}{ PCC, aPCC and recombinant FVIla } \\
\hline
\end{tabular}

Notes: *Activated charcoal may be useful in apixaban and dabigatran overdose if administered early, especially within 2-3 hours of ingestion; §dabigatran has low protein binding and can be dialyzed unlike rivaroxaban and apixaban; ${ }^{\propto}$ limited data but inexpensive and favorable safety profile.

Abbreviations: aPCC, activated prothrombin complex concentrate; FVIla, recombinant factor VII activated; PCC, prothrombin complex concentrate; IV, intravenous.

described in case reports. These products were originally designed for patients with specific factor deficiencies, such as in hemophilia. Their utility in anticoagulation reversal and achieving hemostasis has been better described with warfarin. ${ }^{28,29}$ Their efficacy in NOAC-associated bleeding, however, has not been validated by large randomized controlled trials. The following formulations are available:

- Inactivated PCC

o three factor: FII, FIX, FX

$\circ$ four factor: FII, FVII, FIX, FX

- Activated PCCs

- four factor: activated factor VII, small amounts of FII, FVII, FIX, and FX

- Recombinant FVIIa

- Activated FVII

Studies of PCC in reversal of NOACs have been limited to animal models and small healthy subject studies. Outcomes have largely been centered on the following hemostatic laboratory parameters: anti-factor Xa activity, $\mathrm{INR} /$ prothrombin time (PT), and endogenous thrombin potential-area under the curve.$^{30}$ Evidence that correction of laboratory values translates to clinically significant hemostasis, however, is lacking. Importantly, these complexes do not come without thrombotic risk. These agents are also expensive. Presently, their use is on a case-bycase basis.

Among the PCCs, four-factor PCC in patients on rivaroxaban has the most evidence. One small trial $(n=12)$ reported normalization of PT upon administration of fourfactor PCC in patients anticoagulated with rivaroxaban for 2 days. In patients anticoagulated with dabigatran, PCC did not normalize coagulation studies (PT, ECT, and TT). ${ }^{31}$ Four-factor PCC may also be effective in edoxaban. In a larger randomized controlled trial $(\mathrm{n}=110), 50 \mathrm{IU} / \mathrm{kg}$ 4F-PCC completely reversed effects of edoxaban on bleeding duration and endogenous thrombin potential following punch biopsy. ${ }^{30}$ Factor-three PCC may have a greater effect on thrombin generation but lesser effect on PT compared to four-factor PCC. ${ }^{32}$

Of the factor complexes, activated prothrombin complex concentrates, an activated PCC available as factor eight inhibitor bypassing activity (FEIBA) in the US, has the most promising results in preclinical studies. In a randomized cross-over ex vivo study $(\mathrm{n}=10)$, FEIBA corrected all parameters altered by rivaroxaban administration: endogenous thrombin potential, lag-time, and time to peak. In patients who were administered dabigatran, recombinant FVIIa, and FEIBA corrected dabigatran-mediated prolonged lag-time but not endogenous thrombin potential-area under the curve. ${ }^{33}$

Lastly, the antidotes to the oral direct thrombin inhibitor and factor Xa inhibitors are in development, which will be discussed in the next section. These bind and inactivate the drug's site of action or the drug itself. It is important to note that not all bleeding patients require NOAC reversal. Control of bleeding can be achieved in patients with major bleeding with previously described supportive therapies and general management strategies. The use of specific NOAC reversal agents will likely be restricted to cases with life-threatening bleeding or the need for emergent surgery. Antidotes may even be used for reversal of anticoagulation prior to elective procedures. One antidote, ciraparantag, will also pursue this application. In the clinical setting, this will likely be contingent on cost, indication for anticoagulation, and convenience of bridging therapies.

\section{Specific antidotes/management strategies}

Three antidotes are currently in development for NOAC reversal (Table 2):

- Ciraparantag (PER977): direct thrombin inhibitors, factor Xa inhibitors, and heparins

- Andexanet alfa (AnXa, PRT064445): factor Xa inhibitors

- Idarucizumab (aDabi-Fab, BI 655075): ${ }^{34}$ dabigatran 


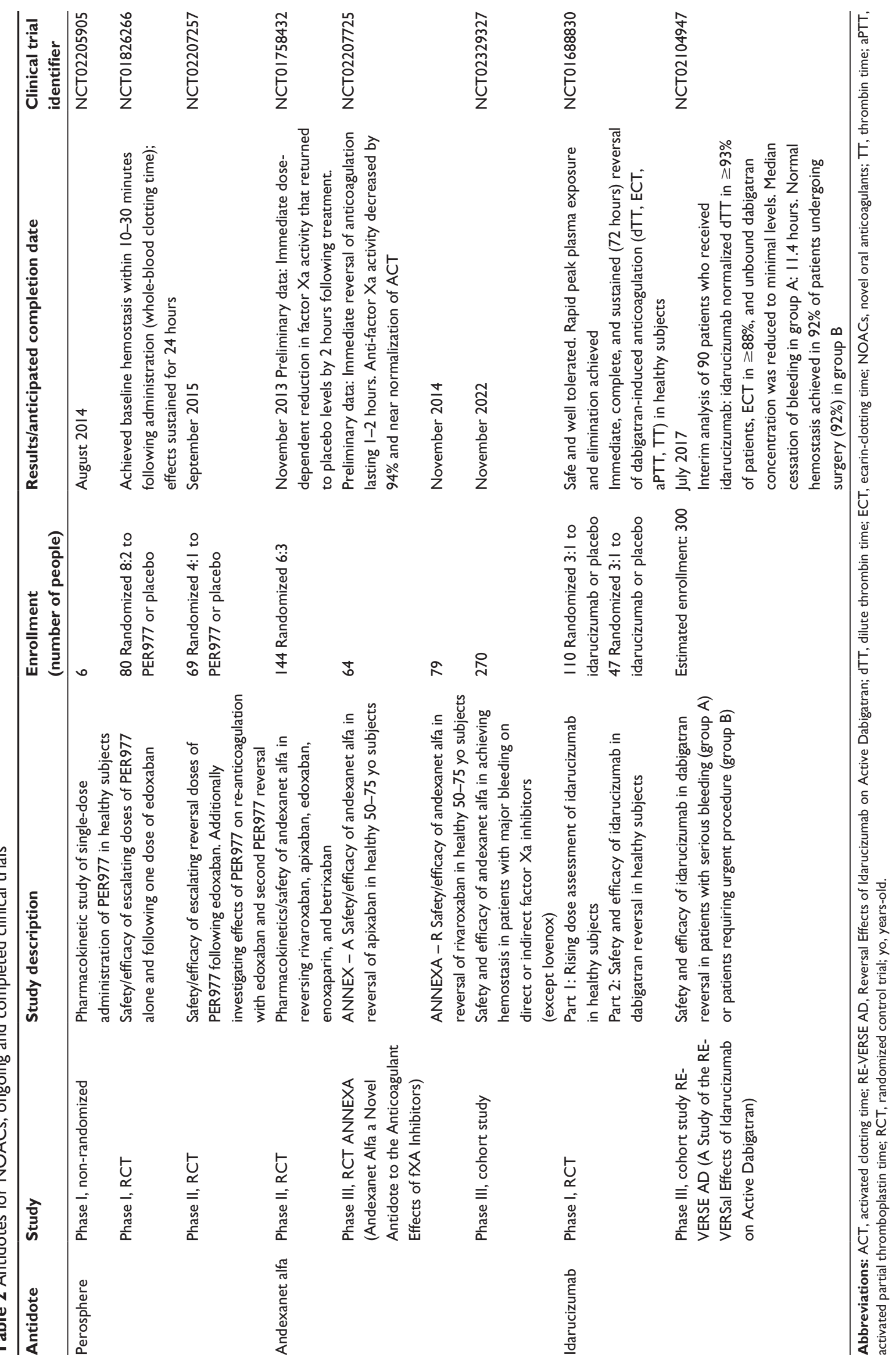


Andexanet alfa and idarucizumab are currently undergoing Phase III clinical trial investigations. Idarucizumab was US FDA approved in October 2015. ${ }^{35}$ Andexanet alfa is undergoing review for FDA approval with "Breakthrough Therapy" designation. ${ }^{35,36}$ Figure 1 depicts the site of action of anticoagulants and their reversal agents in development.

\section{Ciraparantag/PER977: NOACs and heparin antidote}

Ciraparantag (PER977, Perosphere, Danbury, CT, USA) is a small synthetic and cationic molecule that binds direct $\mathrm{Xa}$ inhibitors, direct thrombin inhibitors, and unfractionated and low molecular weight heparin (LMWH) through non-covalent hydrogen bonds and charge--charge interactions (Table 3). There is little published data on the exact mechanism of the molecule. In rat-tail transection bleeding assays, PER977 demonstrated reversal of anticoagulants with thromboelastography studies and reduced bleeding. Edoxaban required the smallest dose for full reversal. In ex vivo human blood studies, PER977 reversed rivaroxaban or apixaban in a dose-dependent fashion, which was quantified by measuring anti-Xa activity. ${ }^{37}$ No procoagulant effects were observed in these studies.

In a preliminary study involving 80 healthy patients, PER977 (100-300 mg IV) was administered to subjects 3 hours after receiving edoxaban (60 mg oral). Anticoagulation reversal was monitored with whole-blood clotting time, which correlated with edoxaban plasma concentration. PER977 restored baseline hemostasis within 10-30 minutes and effects were sustained for 24 hours. ${ }^{38}$ Phase II trials investigating reversal of edoxaban with escalating doses of PER977 are underway. Plans for Phase III trials with edoxaban have also been announced. ${ }^{39}$

Possible procoagulant effects of PER977 have been questioned. PER977 was not found to reverse oral factor Xa inhibitors in human plasma, but instead exhibited possible human platelet activation potentiation. This however was in the absence of signs of platelet aggregation. ${ }^{40}$

\section{Andexanet alfa: factor $\mathbf{X a}$ inhibitor antidote}

Andexanet alfa (PRT064445, Portola Pharmaceuticals, San Francisco, CA, USA) is a recombinant and an inactivated form of factor $\mathrm{Xa}$ engineered as a universal antidote to factor Xa inhibitors. It also binds LMWH and fondaparinux-activated antithrombin III, which act as indirect $\mathrm{Xa}$ inhibitors (Table 4). ${ }^{41}$ Andexanet alfa acts as a decoy factor that binds with high affinity to factor $\mathrm{Xa}$ inhibitors, thus increasing its plasma concentration and reducing its unbound concentration. Though andexanet mimics factor Xa, it does not have procoagulant catalytic activity due to a serine to alanine mutation in the protease catalytic triad. It does not interact with any plasma coagulation proteins, apart from tissue factor pathway inhibitor (TFPI).

Promising ex vivo and animal studies have allowed for the initiation of human clinical trials. ${ }^{42}$ In ex vivo clotting assays, andexanet alfa completely reversed rivaroxaban as quantified by anti-factor Xa activity and PT. In rat models, andexanet normalized INR after infusion with rivaroxaban, apixaban, and betrixaban. The significance of normalized INR was confirmed with restoration of hemostasis by andexanet alfa in rabbits treated with rivaroxaban. In rats anticoagulated by indirect factor Xa inhibitors, enoxaparin (LMWH) and fondaparinux, andexanet alfa completely stopped blood loss after tail transection.

Four separate Phase II randomized, placebo-controlled trials have characterized andexanet alfa's efficacy in a partial reversal of apixaban, rivaroxaban, edoxaban, and enoxaparin. In a small rivaroxaban trial, patients received rivaroxaban $20 \mathrm{mg}$ daily for 6 days followed by escalating doses of andexanet infusion. In those dosed 210 and $420 \mathrm{mg}$, factor $\mathrm{Xa}$ activity was immediately reduced in a dose-dependent manner by $20 \%$ and $53 \%$, respectively. Following termination of infusion, factor Xa activity returned to placebo levels by 2 hours following treatment. No serious adverse events were reported. $^{42}$

Currently Phase III ANNEXA trials (Andexanet Alfa a Novel Antidote to the Anticoagulant Effects of fXA Inhibitors) are testing the safety and efficacy of andexanet alfa in healthy subjects anticoagulated with apixaban (ANNEXAA) and rivaroxaban (ANNEXA-R). Preliminary data in $\mathrm{n}=33$ (treatment $\mathrm{n}=24$ ) showed that an andexanet alfa bolus reduced apixaban-mediated anticoagulation immediately. Anti-factor Xa activity decreased by $94 \%(P<0.0001)$, and near normalization of coagulation (activated clotting time [ACT]) lasted 1-2 hours. ${ }^{43}$ In part two of this phase, a $400 \mathrm{mg}$ IV bolus followed by a 2-hour infusion was evaluated for efficacy in reduction of the plasma-free fraction of apixaban and normalization of thrombin generation and sustained effect. Complete results from these Phase III studies are anticipated. ${ }^{44}$

Although procoagulant adverse effects have not been observed clinically, andexanet's binding of TFPI deserves further investigation. In enzymatic assays, andexanet bound 


\section{Novel oral anticoagulants and their reversal agents}

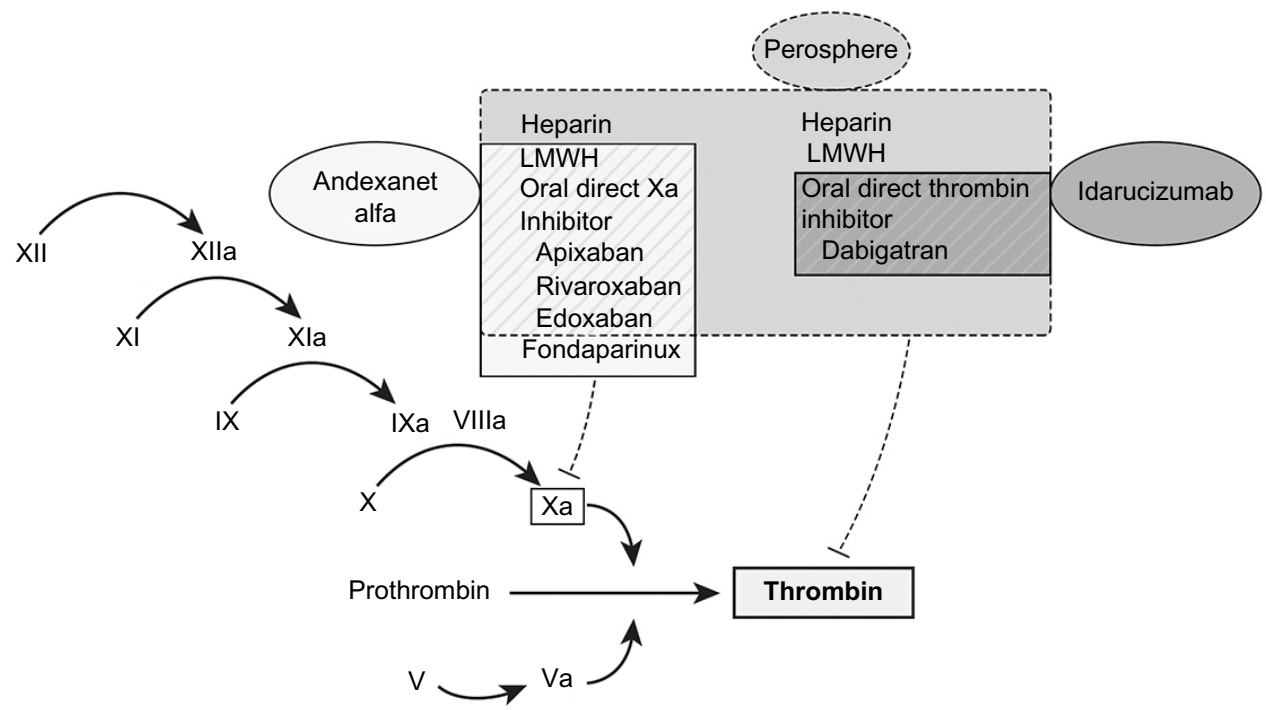

Figure I Coagulation cascade depicting site of action of anticoagulants and reversal agents.

Notes: Dark gray: Idarucizumab - potential reversal agent for direct thrombin inhibitor (dabigatran). Light gray: Perosphere - potential reversal agents for drugs in dashed box. White: Andexanet alfa - potential reversal agent for drugs in solid box.

Abbreviation: LMWH, low molecular weight heparin.

TFPI with similar affinity as that of factor Xa. Unlike the factor X-TFPI complex, the andexanet-TFPI complex does not have enzymatic inhibitory factor $X$ activity. When administered alone, andexanet-TFPI binding did not increase thrombin generation. However, in the pres-

Table 3 Ciraparantag - a universal reversal agent undergoing FDA review

\begin{tabular}{ll}
\hline Ciraparantag (PER977) at a glance \\
\hline Mechanism of action & $\begin{array}{l}\text { Universal reversal agent } \\
\text { Synthetic molecule binds: } \\
\text { Direct Xa inhibitors (apixaban, } \\
\text { rivaroxaban, and edoxaban) } \\
\text { Direct thrombin inhibitors (dabigatran) } \\
\text { Unfractionated and low molecular weight } \\
\text { heparin } \\
\text { Single I00 mg IV dose* } \\
\text { 30 minutes: restoration of WBCT and mean } \\
\text { fibrin-fibrin diameter** } \\
\text { Mild perioral and facial flushing, dysgeusia** } \\
\text { PT remains elevated } \\
\text { Dime to effect } \\
\text { Dos not appear to be sensitive marker for } \\
\text { PER977-mediated anticoagulation reversal* } \\
\text { No prothrombotic effect }{ }^{\S} \\
\text { Life threatening hemorrhage } \\
\text { Emergent surgery } \\
\text { Elective procedures to minimize time off } \\
\text { anticoagulation }\end{array}$ \\
\hline
\end{tabular}

Notes: *Dose being investigated in Phase II trial; **data from Laulicht et al; ${ }^{50}{ }^{\S}$ data from Ansell et al. ${ }^{38}$

Abbreviations: FDA, Food and Drug Administration; PT, prothrombin time; WBCT, whole-blood clotting time; IV, intravenous. ence of rivaroxaban, the andexanet-TFPI interaction led to enhanced thrombin generation. ${ }^{45}$

\section{Idarucizumab: dabigatran antidote} Idarucizumab (aDabi-Fab, BI 655075, Boehringer Ingelheim, Ingelheim am Rhein, Germany) is a humanized mouse monoclonal antibody fragment that binds dabigatran and reverses its anticoagulant effects. Idarucizumab has an extremely high affinity for dabigatran (nearly 350 times stronger than dabigatran's affinity for thrombin), and is able to reverse dabigatran's anticoagulant effects at a 1:1 stoichiometric ratio. ${ }^{46}$ Its mechanism of action is analogous to that of the digoxin antibody fragment antidote (Table 5).

Table 4 Andexanet alfa (PRT064445) - a factor Xa inhibitor antidote undergoing FDA review

\begin{tabular}{|c|c|}
\hline \multicolumn{2}{|c|}{ Andexanet at a glance } \\
\hline Mechanism of action & $\begin{array}{l}\text { Recombinant and inactivated form of factor Xa } \\
\text { Binds factor Xa inhibitors: apixaban, } \\
\text { rivaroxaban, and edoxaban }\end{array}$ \\
\hline Proposed dose & $400 \mathrm{mg}$ IV bolus \pm 2 hours infusion at $4 \mathrm{mg} / \mathrm{min}^{*}$ \\
\hline Time to effect & $\begin{array}{l}2 \text { minutes: } 94 \% \text { decrease in anti } X_{\text {Xa activity }}^{\S} \\
\text { Effects of bolus last I-2 hours }\end{array}$ \\
\hline Adverse effects & $\begin{array}{l}\text { No known prothrombotic effect - tissue factor } \\
\text { pathway inhibitor interaction deserves further } \\
\text { investigation }\end{array}$ \\
\hline Possible indications & $\begin{array}{l}\text { Life-threatening hemorrhage } \\
\text { Emergent surgery }\end{array}$ \\
\hline
\end{tabular}

Notes: *Dose currently being investigated in Phase III, part 2 trial; §data from Crowther et al. ${ }^{44}$

Abbreviation: FDA, Food and Drug Administration; IV, intravenous. 


\section{Pharmacodynamics and pharmacokinetics}

Idarucizumab is administered by intravenous infusion. Idarucizumab binds both free and thrombin-bound dabigatran with a rapid on-rate and slow off-rate. The bound complex is eliminated predominantly by renal excretion. Idarucizumab is distributed solely within the intravascular space, while dabigatran distributes to body tissues as well. Idarucizumab continues to draw dabigatran from the extravascular spaces into the intravascular space and eliminates it. In Phase I studies, peak idarucizumab concentrations occurred shortly after completion of intravenous infusion. ${ }^{47}$ The half-life of idarucizumab is $\sim 45$ minutes.

Though idarucizumab shares structural similarities with thrombin and mimics its binding of dabigatran, it does not have any prothrombotic activity. In Phase I studies, coagulation parameters were unchanged across a wide range of idarucizumab doses. In a Phase I human study, idarucizumab was safe and effective in the reversal of dabigatran anticoagulant activity. ${ }^{48}$

Dabigatran activity and reversal of its anticoagulant activity by idarucizumab was best assessed by measuring dTT and ECT. ${ }^{47}$ These assays best correlated with unbound dabigatran concentration, which was a rough surrogate of pharmacologically active dabigatran. Dabigatran-induced elevations in dTT and ECT were reversed by idarucizumab, and the reversal was sustained at 72 hours for doses greater than $2 \mathrm{~g}$. Idarucizumab resulted in a decrease in unbound dabigatran concentration in plasma to lower limits of detection.

Table 5 Idarucizumab - a direct thrombin (dabigatran) inhibitor undergoing FDA review

\begin{tabular}{ll}
\hline Idarucizumab at a glance & \\
\hline Mechanism of action & Binds free and thrombin-bound dabigatran \\
& Renal elimination of complex \\
Dose & Two $2.5 \mathrm{~g} 50 \mathrm{~mL}$ bolus IV infusions within \\
& I5 minutes: total $5 \mathrm{~g}^{*}$ \\
Half-life & 45 minutes, may require repeat dosing* \\
Time to effect & Normalization of dTT and ECT minutes \\
& after infusion* \\
& Median time to cessation of bleeding: \\
& II.4 hours* \\
Adverse effects & Headache, nasopharyngitis, back pain, skin \\
& irritation - similar to placebo§ \\
Possible indications & No prothrombotic effect \\
& Life-threatening hemorrhage \\
& Emergent surgery \\
& Dabigatran overdose
\end{tabular}

Notes: *Data from Pollack et al: ${ }^{49}$ interim analysis of Phase III study; ${ }^{\S}$ data from Glund et al: ${ }^{47,48}$ Phase I studies.

Abbreviations: dTT, dilute thrombin time; FDA, Food and Drug Administration; ECT, ecarin-clotting time; IV, intravenous.

\section{Phase III data}

A Phase III clinical trial, RE-VERSE AD, is currently evaluating the safety and efficacy of idarucizumab for dabigatran reversal in patients who either A) have life-threatening bleeding judged to require a reversal agent by the treating clinician or B) require an emergent procedure within 8 hours (NCT02104947). An interim analysis of 90 patients (51 patients in group A, 39 in group B) was reported earlier this year. ${ }^{49}$ This is a cohort study, as a randomized trial was deemed unethical due to non-availability of a proven alternative to idarucizumab. Atrial fibrillation was the indication for anticoagulation in $96 \%$ patients. At baseline, 68 patients had elevated dTT and 81 had elevated ECT. Notably patients with normal clotting tests at baseline had better renal function, reflective of dabigatran clearance between patients with normal renal function.

A $5 \mathrm{~g}$ intravenous infusion of idarucizumab was the dose used in this study, which was calculated based on the 99th percentile of total body dabigatran concentration from the Randomised Evaluation of Long-Term Anticoagulation Therapy (RE-LY) study ${ }^{44}$ Idarucizumab resulted in normalization of dTT in $98 \%$ patients in group A and $93 \%$ in group B within minutes. The ECT was normalized in $89 \%$ patients in group A and $88 \%$ in group B. Unbound dabigatran concentration was reduced to minimal levels in all except one patient after minutes of idarucizumab administration.

The median time for cessation of bleeding in group A patients was 11.4 hours. Out of 39 patients in group B, 36 underwent surgery and normal intraoperative hemostasis was present in $92 \%$ of these patients. At 24 hours, the concentration of unbound dabigatran increased, and coagulation parameters were abnormal ( $10 \%$ and $19 \%$ for dTT; $28 \%$ and $46 \%$ for ECT for group A and B, respectively). This is consistent with the short half-life of idarucizumab (45 minutes), and may represent diffusion of extravascular dabigatran into the intravascular space. The authors acknowledge the uncertainty of additional idarucizumab dosing in such patients.

\section{Safety and adverse events}

There were no differences between adverse events among patients receiving idarucizumab or placebo in Phase I studies. Headache, nasopharyngitis, back pain, and skin irritation were the most frequently reported, with similar symptoms reported in patients receiving placebo. No thrombotic complications occurred among idarucizumab patients in Phase I studies. Pre-existing antibodies reactive to idarucizumab were present in $12 \%$ patients, but no adverse events consistent with immunologic reactions were observed. 
In the interim analysis of the Phase III study, thrombotic events occurred in five patients. ${ }^{49}$ None of the patients were receiving anticoagulant therapy at the time of thrombotic event. In the absence of a placebo group, it is not possible to link these events to idarucizumab administration. The data from Phase I studies are reassuring with regard to the safety of idarucizumab. Postmarket surveillance will be vital to identify currently unknown adverse events.

\section{Conclusion}

NOACs offer a safe, effective, and convenient alternative to warfarin for anticoagulation in several conditions. Most bleeding episodes can be managed by cessation of anticoagulation and supportive measures alone. Specific antidotes for NOACs are under development, and idarucizumab has been the first to be approved by the US FDA. These agents will likely be used in patients with life-threatening bleeding such as intracranial hemorrhage, retroperitoneal bleeding, and in patients requiring emergent surgery.

\section{Disclosure}

The authors report no conflicts of interest in this work.

\section{References}

1. Connolly SJ, Ezekowitz MD, Yusuf S, et al. Dabigatran versus warfarin in patients with atrial fibrillation. N Engl J Med. 2009; 361(12):1139-1151.

2. Granger $\mathrm{CB}$, Alexander JH, McMurray JJ, et al. Apixaban versus warfarin in patients with atrial fibrillation. $N$ Engl $J$ Med. 2011; 365(11):981-992.

3. Patel MR, Mahaffey KW, Garg J, et al. Rivaroxaban versus warfarin in nonvalvular atrial fibrillation. $N$ Engl J Med. 2011;365(10):883-891.

4. Ageno W, Gallus AS, Wittkowsky A, Crowther M, Hylek EM, Palareti G. Oral anticoagulant therapy: antithrombotic therapy and prevention of thrombosis, 9th ed: American College of chest physicians evidence-based clinical practice guidelines. Chest. 2012; 141(2 Suppl):e44S-e88S.

5. Fanola CL. Current and emerging strategies in the management of venous thromboembolism: benefit-risk assessment of dabigatran. Vasc Health Risk Manag. 2015;11:271-282.

6. Ment J. Direct oral anticoagulants: key considerations for use to prevent stroke in patients with nonvalvular atrial fibrillation. Vasc Health Risk Manag. 2015;11:317-332.

7. Giugliano RP, Ruff CT, Braunwald E, et al. Edoxaban versus warfarin in patients with atrial fibrillation. $N$ Engl J Med. 2013;369(22): 2093-2104.

8. Fuji T, Fujita S, Kawai Y, et al. Efficacy and safety of edoxaban versus enoxaparin for the prevention of venous thromboembolism following total hip arthroplasty: STARS J-V. Thromb J. 2015;13:27.

9. Fuji T, Wang CJ, Fujita S, et al. Safety and efficacy of edoxaban, an oral factor Xa inhibitor, versus enoxaparin for thromboprophylaxis after total knee arthroplasty: the STARS E-3 trial. Thromb Res. 2014;134(6):1198-1204.

10. NICE. Edoxaban for Treating and Preventing Deep Vein Thrombosis and Pulmonary Embolism. London: National Institute for Health and Care Excellence. Available from: https://www.nice.org.uk/guidance/ ta354. Accessed 2015.
11. Lixiana Edoxaban. Available from: http://www.ema.europa.eu/ema/ index.jsp?curl=pages/medicines/human/medicines/002629/human med_001874.jsp\&mid=WC0b01ac058001d124. Accessed October 24, 2015.

12. Chan NC, Bhagirath V, Eikelboom JW. Profile of betrixaban and its potential in the prevention and treatment of venous thromboembolism. Vasc Health Risk Manag. 2015;11:343-351.

13. Blann A. Nonvitamin K antagonist oral anticoagulants (NOACs): the tide continues to come in. Vasc Health Risk Manag. 2015;11: 489-492.

14. Ruff CT, Giugliano RP, Braunwald E, et al. Comparison of the efficacy and safety of new oral anticoagulants with warfarin in patients with atrial fibrillation: a meta-analysis of randomised trials. Lancet. 2014; 383(9921):955-962.

15. Chai-Adisaksopha $\mathrm{C}$, Crowther $\mathrm{M}$, Isayama $\mathrm{T}$, Lim W. The impact of bleeding complications in patients receiving target-specific oral anticoagulants: a systematic review and meta-analysis. Blood. 2014;124(15):2450-2458.

16. Kakkos SK, Kirkilesis GI, Tsolakis IA. Editor's choice - efficacy and safety of the new oral anticoagulants dabigatran, rivaroxaban, apixaban, and edoxaban in the treatment and secondary prevention of venous thromboembolism: a systematic review and meta-analysis of phase III trials. Eur J Vasc Endovasc Surg. 2014;48(5):565-575.

17. Weitz JI, Pollack CV Jr. Practical management of bleeding in patients receiving non-vitamin $\mathrm{K}$ antagonist oral anticoagulants. Thromb Haemost. 2015;114(5). Epub 2015 Jul 9.

18. Crowther M, Crowther MA. Antidotes for novel oral anticoagulants: current status and future potential. Arterioscler Thromb Vasc Biol. 2015; $35: 1736-1745$.

19. Ebright J, Mousa SA. Oral anticoagulants and status of antidotes for the reversal of bleeding risk. Clin Appl Thromb Hemost. 2015; 21(2):105-114.

20. Wysokinski WE, McBane RD ii. Periprocedural bridging management of anticoagulation. Circulation. 2012;126(4):486-490.

21. Liotta EM, Levasseur-Franklin KE, Naidech AM. Reversal of the novel oral anticoagulants dabigatran, rivoraxaban, and apixaban. Curr Opin Crit Care. 2015;21(2):127-133.

22. Paikin JS, Hirsh J, Chan NC, Ginsberg JS, Weitz JI, Eikelboom JW. Timing the first post-operative dose of anticoagulants: lessons learned from clinical trials. Chest. 2015;148:587-595.

23. Schulman S, Carrier M, Lee AY, et al. Perioperative management of dabigatran: a prospective cohort study. Circulation. 2015; 132(3):167-173.

24. Pollack CV Jr. Coagulation assessment with the new generation of oral anticoagulants. Emerg Med J. Epub 2015 May 18.

25. Wang X, Mondal S, Wang J, et al. Effect of activated charcoal on apixaban pharmacokinetics in healthy subjects. Am J Cardiovasc Drugs. 2014;14(2):147-154.

26. van Ryn J, Sieger P, Kink-Eiband M, Gansser D, Clemens A. Adsorption of dabigatran etexilate in water or dabigatran in pooled human plasma by activated charcoal in vitro. Blood. 2009;114(22):440-440.

27. Chai-Adisaksopha C, Hillis C, Lim W, Boonyawat K, Moffat K, Crowther M. Hemodialysis for the treatment of dabigatran-associated bleeding: a case report and systematic review. J Thromb Haemost. 2015;13:1790-1798.

28. Sarode R, Milling TJ Jr, Refaai MA, et al. Efficacy and safety of a 4-factor prothrombin complex concentrate in patients on vitamin $\mathrm{K}$ antagonists presenting with major bleeding: a randomized, plasma-controlled, phase IIIb study. Circulation. 2013;128(11): 1234-1243.

29. Pabinger I, Brenner B, Kalina U, Knaub S, Nagy A, Ostermann H. Prothrombin complex concentrate (Beriplex P/N) for emergency anticoagulation reversal: a prospective multinational clinical trial. J Thromb Haemost. 2008;6(4):622-631.

30. Zahir H, Brown KS, Vandell AG, et al. Edoxaban effects on bleeding following punch biopsy and reversal by a 4-factor prothrombin complex concentrate. Circulation. 2015;131(1):82-90. 
31. Eerenberg ES, Kamphuisen PW, Sijpkens MK, Meijers JC, Buller HR, Levi M. Reversal of rivaroxaban and dabigatran by prothrombin complex concentrate: a randomized, placebo-controlled, crossover study in healthy subjects. Circulation. 2011;124(14):1573-1579.

32. Levi M, Moore KT, Castillejos CF, et al. Comparison of three-factor and four-factor prothrombin complex concentrates regarding reversal of the anticoagulant effects of rivaroxaban in healthy volunteers. J Thromb Haemost. 2014;12(9):1428-1436.

33. Marlu R, Hodaj E, Paris A, Albaladejo P, Cracowski JL, Pernod G. Effect of non-specific reversal agents on anticoagulant activity of dabigatran and rivaroxaban: a randomised crossover ex vivo study in healthy volunteers. Thromb Haemost. 2012;108(2):217-224.

34. Idarucizumab. Available from: http://www.fda.gov/Drugs/InformationOnDrugs/ApprovedDrugs/ucm467396.htm. Accessed: November 2, 2015.

35. Boehringer Ingelheim's Investigational Antidote for Pradaxa ${ }^{\circledR}$ (dabigatran etexilate mesylate) receives FDA breakthrough therapy designation. Available from: http://us.boehringer-ingelheim.com/news_events/ press_releases/press_release_archive/2015/4-23-2015-fda-grantspriority-review-boehringer-ingelheims-biologics-license-applicationidarucizumab.html. Accessed October 10, 2015.

36. Portola pharmaceuticals receives breakthrough therapy designation from FDA for andexanet alfa (PRT4445*), investigational factor Xa inhibitor antidote. Available from: http://investors.portola.com/ phoenix.zhtml?c=198136\&p=irol-newsArticle\&ID=1879666. Accessed August 5, 2015.

37. Laulicht B, Bakhru S, Lee C, et al. Small molecule antidote for anticoagulants. Circulation. 2012;126:A11395.

38. Ansell JE, Bakhru SH, Laulicht BE, et al. Use of PER977 to reverse the anticoagulant effect of edoxaban. $N$ Engl J Med. 2014; 371(22):2141-2142.

39. Perosphere and daiichi sankyo enter into a clinical trial collaboration agreement for phase 3 studies of PER977 to investigate reversal of the anticoagulant activity of the investigational factor Xa-inhibitor edoxaban. Available from: http://perosphere.com/content/media/ documents/PerosphereandDaiichiSankyoEnterintoaClinicalTrialAgreementforPhase3.pdf. Accessed October 10, 2015.

40. Lu G, Kotha J, Cardenas JM, et al. In vitro characterization of andexanet alfa (PRT064445), a specific fXa inhibitor antidote versus aripazine (PER977), a non-specific reversal agent. Circulation. 2014;130: A18218.
41. Lu G, DeGuzman FR, Hollenbach SJ, et al. A specific antidote for reversal of anticoagulation by direct and indirect inhibitors of coagulation factor Xa. Nat Med. 2013;19(4):446-451.

42. Crowther M, Vandana M, Michael K, et al. A phase 2 randomized, double-blind, placebo-controlled trial demonstrating reversal of rivaroxaban induced anticoagulation in healthy subjects by andexanet alfa (PRT064445), an antidote for fxa inhibitors (abstract). Blood. 2013;122(21):3636.

43. Crowther M, Levy G, Lu G, et al. ANNEXA-A: a phase 3 randomized, double-blind, placebo-controlled trial, demonstrating reversal of apixaban-induced anticoagulation in older subjects by andexanet alfa (PRT064445), a universal antidote for factor Xa (fXa) inhibitors. Circulation. 2014;130:2105-2126.

44. Crowther M, Gold A, Lu G, et al. ANNEXA (TM)-A PART 2: a phase 3 randomized, double-blind, placebo-controlled trial demonstrating sustained reversal of apixaban-induced anticoagulation in older subjects by andexanet ALFA (PRT064445), a universal antidote for factor XA (FXA) inhibitors. J Thromb Haemost. 2015;13:84-85.

45. Lu G, Lin J, Coffey G, Curnutte JT, Conley PB. Interaction of andexanet alfa, a universal antidote to fXA inhibitors, with tissue factor pathway inhibitor enhances reversal of fXA inhibitor-induced anticoagulation. J Thromb Haemost. 2015;13:634-635.

46. Schiele F, van Ryn J, Canada K, et al. A specific antidote for dabigatran: functional and structural characterization. Blood. 2013; 121(18):3554-3562.

47. Glund S, Stangier J, Schmohl M, et al. Safety, tolerability, and efficacy of idarucizumab for the reversal of the anticoagulant effect of dabigatran in healthy male volunteers: a randomised, placebo-controlled, doubleblind phase 1 trial. Lancet. 2015;386:680-690.

48. Glund S, Moschetti V, Norris S, et al. A randomised study in healthy volunteers to investigate the safety, tolerability and pharmacokinetics of idarucizumab, a specific antidote to dabigatran. Thromb Haemost. 2015;113(5):943-951.

49. Pollack CV Jr, Reilly PA, Eikelboom J, et al. Idarucizumab for dabigatran reversal. N Engl J Med. 2015;373:511-520.

50. Laulicht B, Bakhru S, Steiner S, et al. PER977 (ciraparantag) reverses edoxaban anticoagulation at steady state and has no effect on reanticoagulation at the next scheduled dose. Eur Heart J. 2015;36 (Abstract Supplement)
Vascular Health and Risk Management

\section{Publish your work in this journal}

Vascular Health and Risk Management is an international, peerreviewed journal of therapeutics and risk management, focusing on concise rapid reporting of clinical studies on the processes involved in the maintenance of vascular health; the monitoring, prevention and treatment of vascular disease and its sequelae; and the involvement of

\section{Dovepress}

metabolic disorders, particularly diabetes. This journal is indexed on PubMed Central and MedLine. The manuscript management system is completely online and includes a very quick and fair peer-review system, which is all easy to use. Visit http://www.dovepress.com/ testimonials.php to read real quotes from published authors. 